\title{
Modificação da superfície do poli (éter-éter-cetona)
}

\author{
Surface modification of poly \\ (ether-ether-ketone)
}

Flavia Suzany Ferreira dos Santos ${ }^{1}$, Valéria Pereira Ferreira ${ }^{1}$,

\author{
${ }^{1,}$ Laboratório de Avaliação e Desenvolvimento de Biomateriais do Nordeste - CERTBIO - UEMA/UFCG. Aprígio Ve- \\ loso 882, Bodocongó. CEP 58429 - 900, Campina Grande, PB \\ e-mail: suzanyfs@globo.com; valeriap.ferreira@hotmail.com; mayelli.dantas@gmail.com; marcus.fook@pq.cnpq.br
}

\section{RESUMO}

O poli (éter-éter-cetona) - (PEEK) é conhecido como um biomaterial alternativo para a substituição de materiais metálicos implantáveis. No entanto, para aplicações biomédicas ele é biologicamente inerte, impedindo uma boa interação entre o implante e os tecidos ósseos adjacentes, dificultando a sua aplicação. Uma maneira de superar o caráter inerte do PEEK é a modificação física da sua superfície pelo método de lixiviação de partículas. Dessa forma, este trabalho teve como objetivo desenvolver e caracterizar estruturas de PEEK com superfície modificada. Para a preparação das estruturas utilizou-se a técnica de deposição de uma camada de $\mathrm{NaCl}$ sobre o polímero. Esse material foi submetido a uma carga de 6 toneladas seguido de tratamento térmico a $390^{\circ} \mathrm{C}$ durante três diferentes tempos. Após o resfriamento, as amostras foram submetidas ao processo de lixiviação de partículas de $\mathrm{NaCl}$. Em seguida, as estruturas foram caracterizadas por Microscopia Ótica, retilineidade e FTIR. O aumento de tempo no tratamento térmico conduziu as amostras a uma melhor conformação e preservação das cavidades presentes na superfície rugosa como consequência de uma melhor fusão do polímero, porém provocou uma menor efetividade na lixiviação das partículas de $\mathrm{NaCl}$. No ensaio de retilineidade, a amostra que apresentou menor rugosidade foi a com maior tempo de tratamento térmico, possivelmente devido a uma maior quantidade de $\mathrm{NaCl}$ retido em sua superfície rugosa. A partir das observações dos espectros, as amostras de PEEK, quando comparadas à amostra controle, apresentaram um comportamento similar, não demonstrando alteração significativa no perfil entre os materiais estudados. Considerando os aspectos de processamento, a técnica desenvolvida nesta pesquisa demonstrou-se efetiva para a obtenção das amostras de PEEK com superfície modificada com diferentes tempos de tratamento térmico.

Palavras-chave: PEEK, modificação de superfície, lixiviação de partículas.

\section{ABSTRACT}

The poly (ether ether ketone) - (PEEK) is known as an alternative biomaterial to replace the well-known successful implantable metallic materials. However, the main issue for PEEK as a biomedical implant it is biologically inert, preventing a good interaction between the implant and the surrounding bone tissue. One way to overcome the inert character of PEEK is the physical surface modification through the particle leaching method. In this way, this article has as goal to develop and to characterize PEEK structures which have modified surface. To prepare these structures it was used a deposition technique where a layer of $\mathrm{NaCl}$ is deposited over the polymer. The material was subjected to the load of 6 tons and heat treatment at $390^{\circ} \mathrm{C}$ in three different times. After cooling at room temperature, the samples were leached by NaCI particles process. Then, the structures were examined by optical microscopy, straightness and FTIR. According to analysis, increasing the heat treatment time results in a better polymer melting which leads better rough surfaces. The surfaces, in general, have better form, better maintaining and better depth recesses. However, increasing the heat treatment time produced a lower leaching effectiveness. In straightness test result, the sample showed less roughness was the sample with longer heat treatment, possibly due to a greater amount of $\mathrm{NaCl}$ retained in its roughened surface. From the observations of the FTIR spectra, samples of PEEK, when compared to the con- 
trol sample showed a similar behavior, showing no significant change in the profile of the studied materials. Considering the process, the technique developed in this article showed to be effective obtaining PEEK samples morphologically modified by different heat treatment time.

Keywords: PEEK, surface modification, leaching.

\section{INTRODUÇÃO}

O desenvolvimento de materiais poliméricos tem impulsionado cada vez mais o uso de polímeros como biomateriais, sendo assim, a sua utilização desde aplicações temporárias até aplicações de caráter permanente. Atualmente o polímero poli (éter-éter-cetona) (PEEK) é bastante conhecido como um biomaterial alternativo para materiais metálicos implantáveis $[1,2]$.

O PEEK pertence à família dos polímeros poliariletercetona (PEAK), e foi originalmente desenvolvido no final de 1970 pela indústria aeroespacial nos Estados Unidos da América (EUA). Historicamente, a disponibilidade do PEEK chegou num momento em que havia um crescente interesse no desenvolvimento de hastes e placas de fixação de fratura, com rigidezes comparáveis com o osso. No final de 1990 o PEEK foi comercializado e, posteriormente aceito pela indústria de dispositivos médicos como o principal candidato termoplástico de alto desempenho para substituir os componentes de implantes de metal, especialmente em ortopedia e traumatologia [3, 4].

O PEEK acabou ganhando aceitação generalizada como um polímero resistente, biocompativel, e com propriedades mecânicas semelhantes às de ossos humanos, em comparação com implantes metálicos tradicionais há menos risco do efeito blindagem causado por incompatibilidade da rigidez do material entre o implante e o tecido biológico. Este polímero também possui características radiolúcidas, ou seja, os implantes fabricados a partir do PEEK são compatíveis com diagnósticos de imagem, ao contrário dos implantes metálicos. A sua resistência ao calor e compatibilidade química com várias técnicas de esterilização, juntamente com técnicas de moldagem de baixo custo fazem do PEEK um material prático e econômico para a fabricação de dispositivos médicos $[3,4,5,6,7,8,9]$.

O PEEK trata-se de uma resina termoplástica, semicristalina de cadeia linear aromática interconectadas por grupos cetona $(\mathrm{C}=\mathrm{O})$ e éter $(-\mathrm{O}-)$. A sua estrutura química lhe confere estabilidade a altas temperaturas, e a sua elevada resistência química é complementada pela sua elevada resistência mecânica, produto de seu caráter semicristalino e da alta rigidez molecular de sua unidade de repetição $[3,4,10,11]$.

As atividades mais importantes no uso do PEEK em implantes baseiam-se em aplicações ortopédicas para implantes estruturais. Outros desenvolvimentos estão em estudos em aplicações cardiovasculares, como válvulas cardíacas e marca-passos, na área da odontologia, implantes para fixação de dentes artificiais [4, 12]. Experiências clínicas mostraram que o uso do PEEK em seres humanos não revelou até o momento nenhuma preocupação quanto a reações carcinogênicas [13].

Por mais que o PEEK seja biocompatível, quimicamente estável, radiolucente e tenha um módulo elástico semelhante ao do osso humano normal, com relação às aplicações biomédicas, ele é biologicamente inerte, ou seja, não tem uma boa interação entre o implante e os tecidos ósseos adjacentes, dificultando assim sua aplicação na área biomédica. Sem a osseointegração (interação do implante com o osso), os implantes podem afrouxar ou migrar, causando dor, deformidade ou deficiência ao paciente. A energia de superfície relativamente baixa do PEEK também pode limitar a osseointegração, e potencializar a encapsulação fibrosa, diminuindo a cicatrização de feridas nas aplicações que exigem a integração [2, 8, 14, 15]. Algumas estratégias têm sido utilizadas para superar o carácter inerte do PEEK. Uma abordagem é a modificação física da superfície deste polímero pelo método de lixiviação de partículas.

O método de lixiviação de partículas envolve a lixiviação seletiva de um mineral, normalmente o sal de $\mathrm{NaCl}$ ou de um composto orgânico, como sacarose, para gerar os poros. As partículas do sal são incorporadas ao polímero em solução ou moldadas juntamente durante a fundição do polímero por meio de técnicas como compressão, extrusão, injeção. O processo de lixiviação do agente porogênico irá ocorrer após a elimininação do solvente ou resfriamento da mistura, onde as amostras serão imersas em água para promover a lixiviação do sal [16].

Esta modificação física possui como vantagem a restrição da rugosidade apenas na superfície do polímero promovendo assim a osseointegração e mantendo as propriedades mecânicas do material, não alterando o caráter hidrofílico nem hidrofóbico deste material, e propiciando o crescimento celular naquela superfície modificada fisicamente $[1,7]$.

Dessa forma, este trabalho teve como objetivo desenvolver e caracterizar estruturas de PEEK com superfície modificada. 


\section{MATERIAIS E MÉTODOS}

\subsection{Materiais e instrumentos}

Foi utilizado o polímero PEEK em pó fornecido pela empresa Victrex ${ }^{\circledR}$, (tipo de PEEK utilizado: Vicote 702). Cloreto de sódio ( $\mathrm{NaCl})$ P.A, da marca Nuclear.

\subsection{Procedimento experimental}

A matéria prima, PEEK foi seca em estufa a $150^{\circ} \mathrm{C}$ por $3 \mathrm{~h}$. Para a confecção dos corpos de prova foi utilizado um molde metálico com $30 \mathrm{~mm}$ de diâmetro, contendo aproximadamente $1 \mathrm{~g}$ de $\mathrm{NaCl}$ seguido da adição de $2 \mathrm{~g}$ de PEEK. Após este procedimento, o molde preenchido foi levado a prensa hidráulica e submetido a uma carga de 6 toneladas. Após a realização deste procedimento, o molde e a peça compactada foram tratados termicamente a temperatura de $390^{\circ} \mathrm{C}$. Foram confeccionados três diferentes corpos de prova com variação no tempo de tratamento térmico, sendo a amostra $\mathrm{A}(10$ minutos $), \mathrm{B}(25$ minutos), (55 minutos).

Após o resfriamento, as amostras foram submetidas ao processo de lixiviação de partículas, imergindo as mesmas em água para a remoção do $\mathrm{NaCl}$ por $72 \mathrm{~h}$, e assim, ocasionando a modificação da superfície do polímero. O processo de lixiviação das partículas ocorreu nas mesmas condições para todas as amostras. Após este procedimento, as amostras foram secas em estufa a $60^{\circ} \mathrm{C}$ por aproximadamente $2 \mathrm{~h}$ para a realização das caracterizações.

\subsection{Caracterizações das amostras}

\subsubsection{Microscopia ótica (MO)}

Para a avaliação da morfologia da superfície rugosa e secção transversal das amostras foi utilizado um Microscópio Advanced 3D Digital, Hirox - KH7700, que permite reflexão e transmissão com profundidade de campo extendida com medições 2D e 3D, estando acoplado a uma estação de análise de imagens. Para a aplicação desta técnica foram utilizados aumentos que variaram de 40x a 60x, sendo 40x na secção transversal e 60x na superfície rugosa. É importante ressaltar que para a avaliação da MO na secção transversal as amostras foram fraturadas em nitrogênio líquido.

A distribuição do tamanho de cavidades foi caracterizado através das medidas dos diâmetros das cavidades das imagens de MO das amostras A, B e C, utilizando o software de análise ImageJ. Para cada análise, foi medido o diâmetro de pelo menos 80 cavidades.

\subsubsection{Retilineidade}

A avaliação da retilineidade foi realizada em um equipamento de ensaio tridimensional da marca Mitutoyo Europe Gmbh modelo Crysta-Apex S574 com velocidade de operação $5 \mathrm{~mm} / \mathrm{s}$ e alta precisão, associado ao sistema de compensação de temperatura, e ao controle numérico computadorizado. Após a varredura foram registrados os pontos dos picos e vales para que posteriormente fossem traçados os gráficos de rugosidade de cada amostra.

\subsubsection{Espectroscopia na região do infravermelho com transformada de Fourier (FTIR)}

Com a finalidade de identificar as bandas características dos grupos funcionais presentes nas amostras utilizadas nesta pesquisa e também observar se houve alguma alteração no perfil das amostras entre os materiais foi realizada a análise de espectroscopia na Região do Infravermelho com Transformada de Fourier (FTIR) diretamente nas amostras, no equipamento da Perkin Elmer Spectrum 400, onde se utilizou uma faixa de varredura na região do infravermelho médio de 2000 a $600 \mathrm{~cm}^{-1}$. A matéria prima em pó do polímero PEEK foi denominada amostra controle.

\section{RESULTADOS E DISCUSSÃO}

\subsection{Microscopia ótica (MO)}

Na Figura 1 estão apresentadas as micrografias da secção transversal das amostras A, B e C.

Com relação às micrografias da secção transversal das amostras, foi observado que a amostra $\mathrm{A}$ (tempo de tratamento térmico de 10 minutos) possivelmente apresentou um material com características de não fundido, visto que, após o processo de lixiviação de partículas de $\mathrm{NaCl}$ não houve apenas a remoção do $\mathrm{NaCl}$ 
mas também houve o arraste do polímero. Este arraste do polímero foi devido ao tempo não ser suficiente para que ocorresse o processo de fusão do polímero, portanto as partículas da amostra A estavam apenas compactadas, então quando a amostra A entrou em contato com a água no processo de lixiviação isso fez com o que não ocorresse apenas a remoção do $\mathrm{NaCl}$ mas também de uma parte do polímero.

$\mathrm{Na}$ amostra $\mathrm{B}$ com tempo de tratamento térmico de 25 minutos, foi percebida uma mudança significativa entre ela e amostra A. Essa mudança foi devido ao aumento no tempo de tratamento térmico, que foi o que ocasionou a possível fusão do material, fazendo com o que a amostra B apresentasse uma região lisa(sem perfil de rugosidade) e uma região com perfil de rugosidade e pequenas cavidades bastante visíveis que foi possível após a remoção do $\mathrm{NaCl}$ no processo de lixiviação.

Nesta Figura 1 também foi observado à alteração na cor da amostra C. A amostra $\mathrm{C}$ com tempo de tratamento térmico de 55 minutos apresentou uma coloração diferente com relação às outras amostras, possivelmente devido a um processo oxidativo que ocorreu em sua superfície que pode estar relacionado ao tempo prolongado no tratamento térmico.

A degradação oxidativa do PEEK inclui duas reações, cisão de cadeia e reticulação. Jones et al. [17] em seu estudo sobre a cristalização do PEEK por aquecimento durante tempo prolongado a temperatura acima de $400^{\circ} \mathrm{C}$ mostrou que tanto a nucleação como o crescimento de esferulitos são reduzidos, o que implica em uma diminuição da cristalinidade, favorecendo a formação de ligações cruzadas na estrutura. Day et al. [18] mostraram que o processamento de PEEK durante tempos prolongados e temperaturas elevadas sob atmosfera de oxigênio provocam reações oxidativas, que possivelmente são precursoras das reações de reticulação. O estudo experimental de Jonas e Legras [19] revelou que o processamento de PEEK a temperaturas relativamente altas "leva automaticamente a sua degradação". Sob atmosfera de oxigênio a degradação tornase muito rápida, observando aumentos de $30 \%$ após 30 minutos de aquecimento a temperatura de $400^{\circ} \mathrm{C}$.
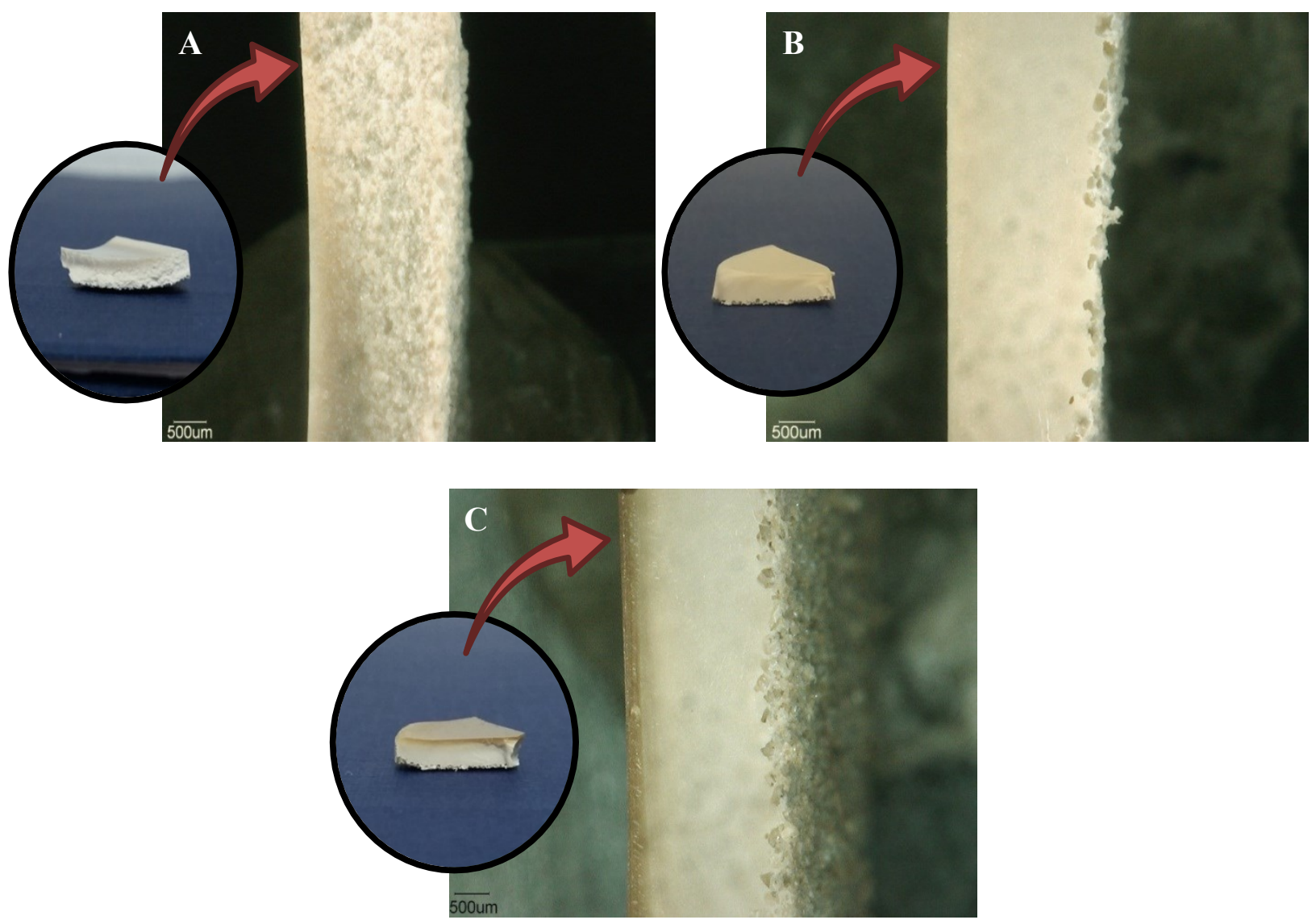

Figura 1: Micrografias referentes à secção transversal com diferentes tempos de tratamento térmico das amostras $\mathrm{A}(10$ minutos $), \mathrm{B}(25$ minutos $)$ e $\mathrm{C}(55$ minutos $)$.

Na Figura 2 estão apresentadas as micrografias da superfície rugosa das amostras A, B e C. Foi observado na amostra $\mathrm{B}$, quando comparada com $\mathrm{A}$, que o aumento no tempo de tratamento térmico conduziu a 
uma melhor conformação e preservação das cavidades presentes na superfície rugosa como consequência de uma melhor fusão do polímero e lixiviação das partículas. Além disto, foi observado que com o aumento excessivo no tempo de tratamento térmico houve uma menor efetividade na lixiviação das partículas de $\mathrm{NaCl}$, como pode ser observado na amostra C. Este aumento no tempo fez com o que ocorresse a fusão do polímero, promovendo uma maior fluidez. Essa maior fluidez provavelmente foi o que ocasionou a percolação do polímero fundido pelo $\mathrm{NaCl}$ com maior facilidade, promovendo o encapsulamento das partículas de $\mathrm{NaCl}$ e dificultando assim na sua remoção no processo de lixiviação.

Kurtz [4] na combinação do método de moldagem por compressão do PEEK e lixiviação de partículas pode ocorrer o encapsulamento das partículas de sal, impedindo a sua completa remoção no processo de lixiviação.
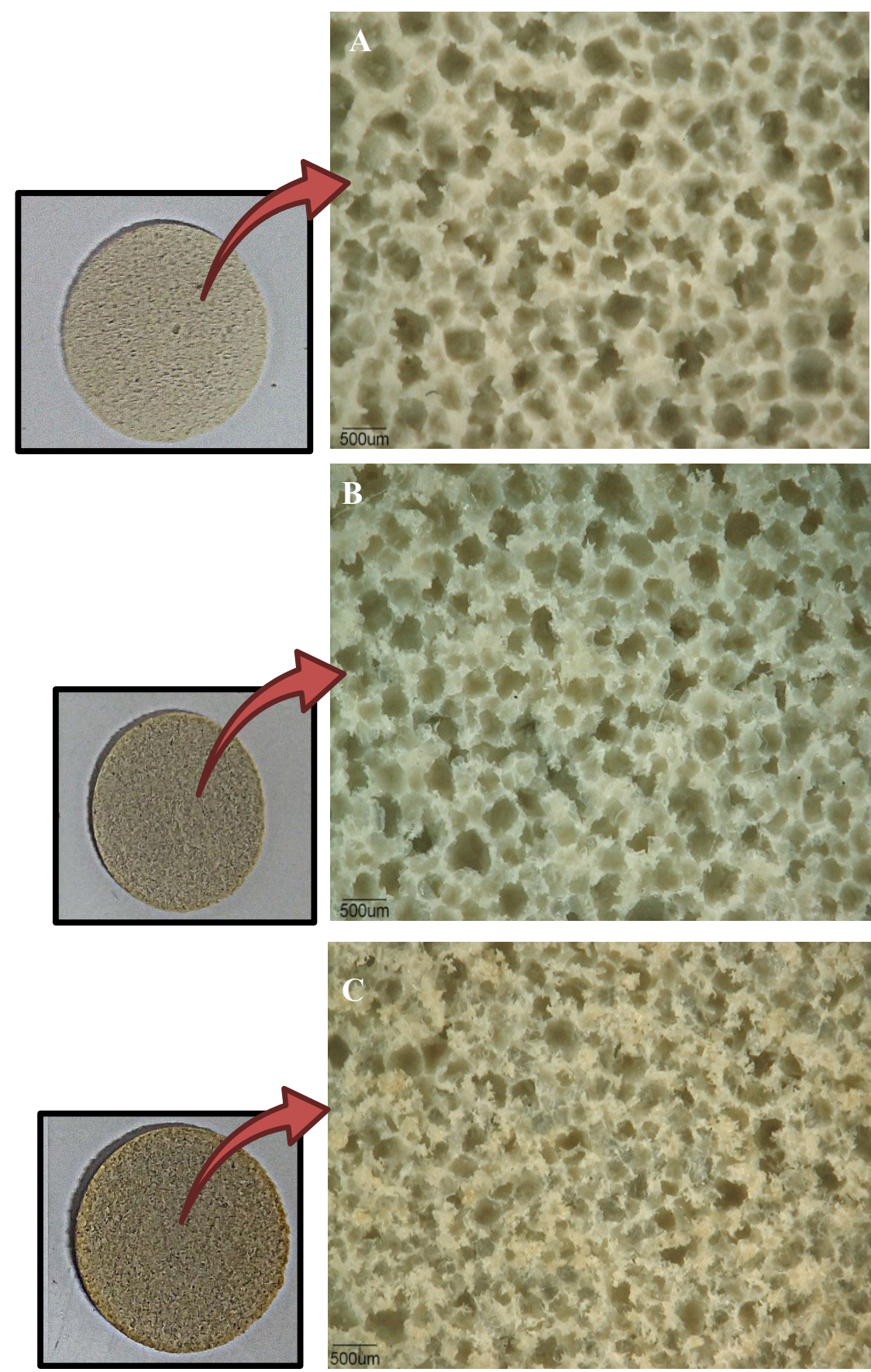

Figura 2: Micrografias referentes à superfície rugosa com diferentes tempos de tratamento térmico das amostras $\mathrm{A}(10$ minutos $), \mathrm{B}(25$ minutos $)$ e $\mathrm{C}(55$ minutos $)$. 
$\mathrm{O}$ mecanismo de lixiviação do $\mathrm{NaCl}$ consiste na solubilização do agente porogênico em água, através de um arraste mecânico dessas partículas. A solubilização deste agente porogênico vai modificar a morfologia do material nos dando um diâmetro médio das cavidades de acordo com a granulometria do $\mathrm{NaCl}$.

O tamanho das partículas do agente porogênico estão na faixa de 180-450 $\mu \mathrm{m}$, com uma média de tamanho de partículas de $314 \mu \mathrm{m}$, e de acordo com a distribuição granulométrica realizada através do histograma (Figura 3) é possível observar que cerca de 78\% das partículas de $\mathrm{NaCl}$ encontram-se na faixa de 200 $400 \mu \mathrm{m}$.

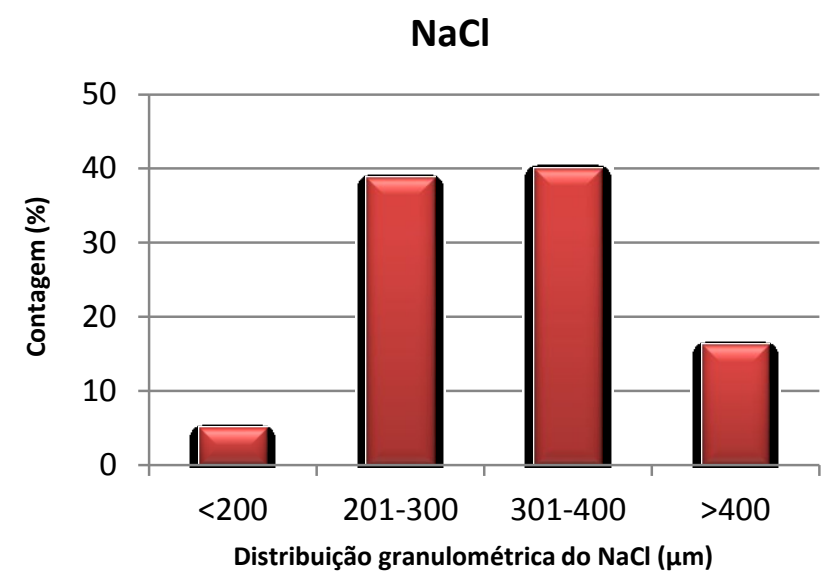

Figura 3: Histograma da distribuição granulométrica do $\mathrm{NaCl}$ medidos com o software ImageJ.

De acordo com o cálculo do diâmetro médio das cavidades foi possível observar que as amostras A, B e C, apresentaram diâmetro médio de $397 \mu \mathrm{m}, 333 \mu \mathrm{m}$ e $234 \mu \mathrm{m}$, respectivamente. Analisando os histogramas apresentados na Figura 4(A), 4(B) e 3(C), podemos perceber que o perfil das amostras estudadas mudou com o aumento no tempo de tratamento térmico, refletindo assim no histograma. Como havia sido mencionado anteriormente, na microscopia ótica, a amostra A com menor tempo, apresentou cavidades maiores devido ao arraste do polímero no processo de lixiviação, isto é possível observar na Figura(A), onde, 46\% das cavidades presentes na amostra possuem diâmetro acima de $400 \mu \mathrm{m}$, em contrapartida, a amostra C foi apresentou uma menor cavidade devido ao encapsulamento do agente porogênico, dificultando a sua remoção no processo de lixiviação, possuindo $83 \%$ de cavidades com tamanhos $<300 \mu \mathrm{m}$ (Figura(C)). Por fim, a morfologia das cavidades da amostra B correlacionou-se de forma confiável ao tamanho das partículas do agente porogênico, onde cerca de $71 \%$ de cavidades apresentaram diâmetro de 200-400 $\mu \mathrm{m}$. Portanto, o mecanismo de lixiviação promoveu a modificação na morfologia da superfície do PEEK.

Amostra A

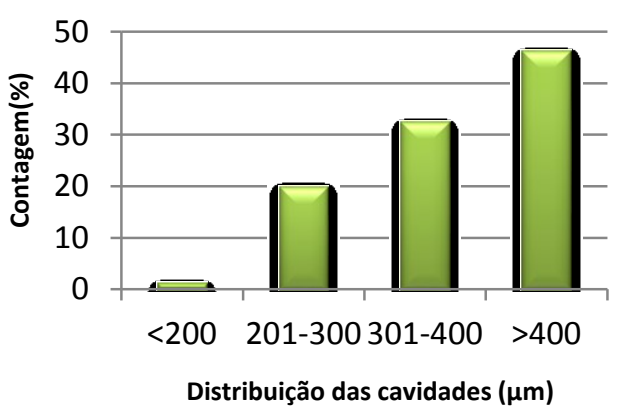

(B)

\section{Amostra B}

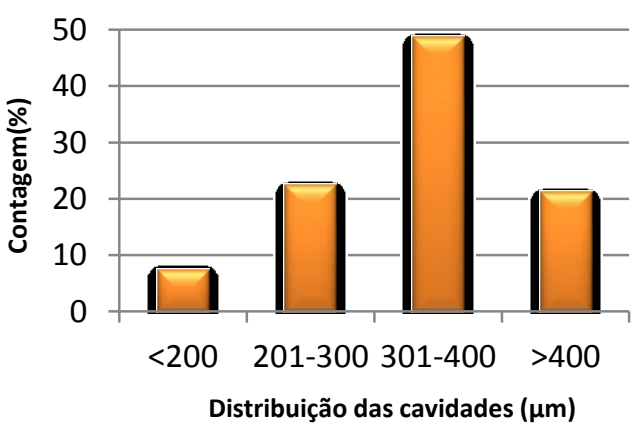

(A) 


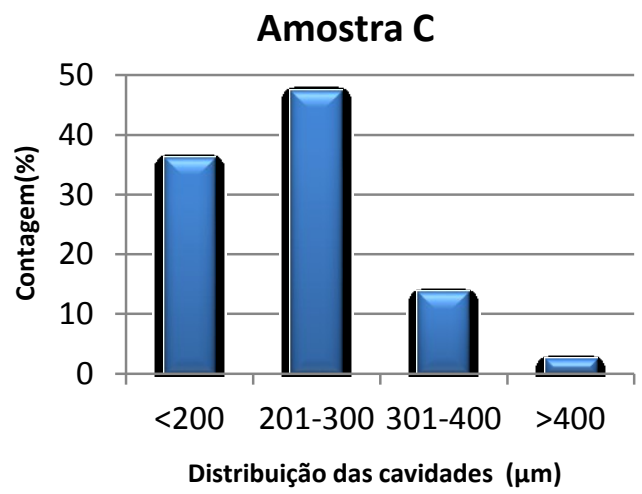

(C)

Figura 4: Histograma da distribuição das cavidades para as amostras A (10 minutos), B(25 minutos) e C(55 minutos), medidos com o software ImageJ.

Os resultados encontrados no presente estudo com relação ao tamanho médio das cavidades nas amostras estão de acordo com a faixa de tamanho médio de poros estimado por Siddiq e Kennedy [1] no qual, os mesmos utilizaram do método de lixiviação de partículas de $\mathrm{NaCl}$ para a produção de estruturas porosas de PEEK, com resultados de $85 \%$ de porosidade e diâmetro médio de poros estimado entre 250 e $350 \mu \mathrm{m}$. Evans et al. [7] investigaram um novo método para criar um material a base de PEEK com a superfície porosa com o objetivo de promover a osseointegração, utilizaram a técnica de extrusão com lixiviação de partículas de $\mathrm{NaCl}$ e alcançaram resultados com tamanho médio de poros de $280 \mu \mathrm{m}$.

O estudo realizado por Whang et al. [20], demonstrou através de experimentos o efeito do tamanho do poro na regeneração de tecidos. Os poros de $5 \mu \mathrm{m}$ permitem a neovascularização, entre $5-15 \mu \mathrm{m}$ o crescimento de fibroblastos, 40-100 $\mu \mathrm{m}$ crescimento da matriz osteóide e 100-350 $\mu \mathrm{m}$ regeneração óssea.

\subsection{Retilineidade}

Para a verificação da superfície rugosa de cada amostra foi utilizado o ensaio de retilineidade. As amostras apresentaram $30 \mathrm{~mm}$ de diâmetro, sendo que a varredura foi realizada em $14 \mathrm{~mm}$ da amostra em 29 pontos com a sua superfície submetida à análise sempre voltada para cima a uma velocidade de varredura de 5,00 $\mathrm{mm} / \mathrm{s}$, aplicadas com limite de tolerâncias de $0,5 \mathrm{~mm}$, estabelecido por metodologia adotada no Laboratório CERTBIO, conforme demonstrado na Figura 5.

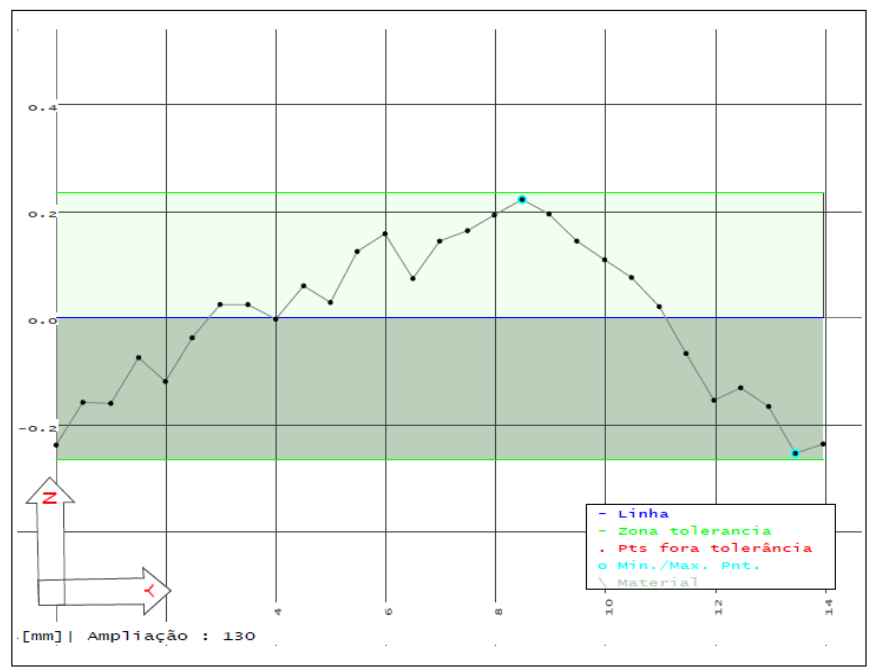




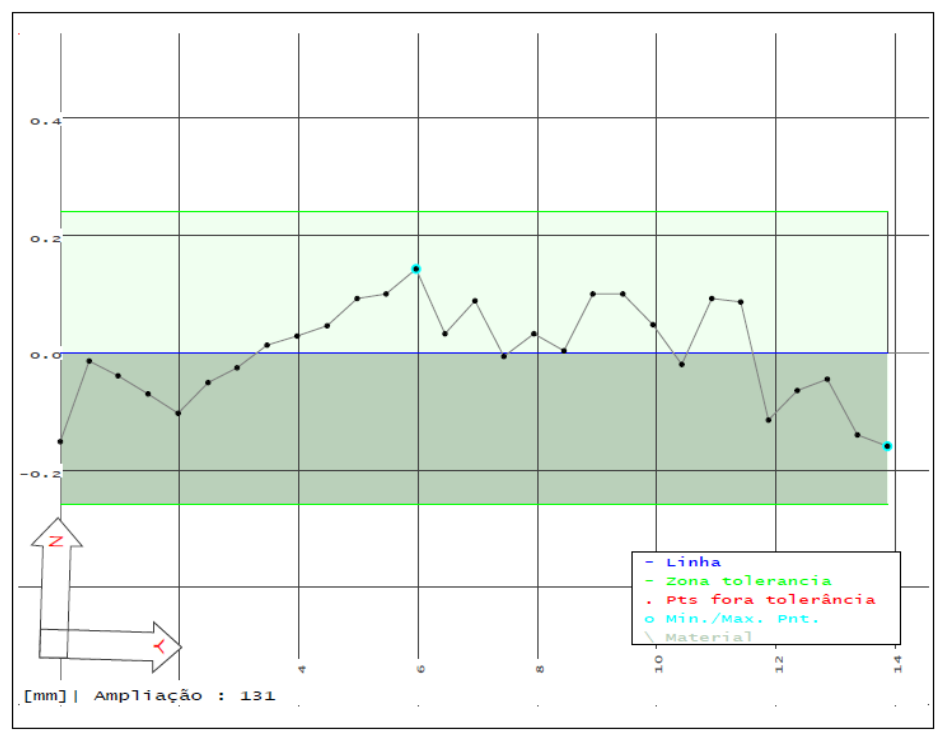

(B)

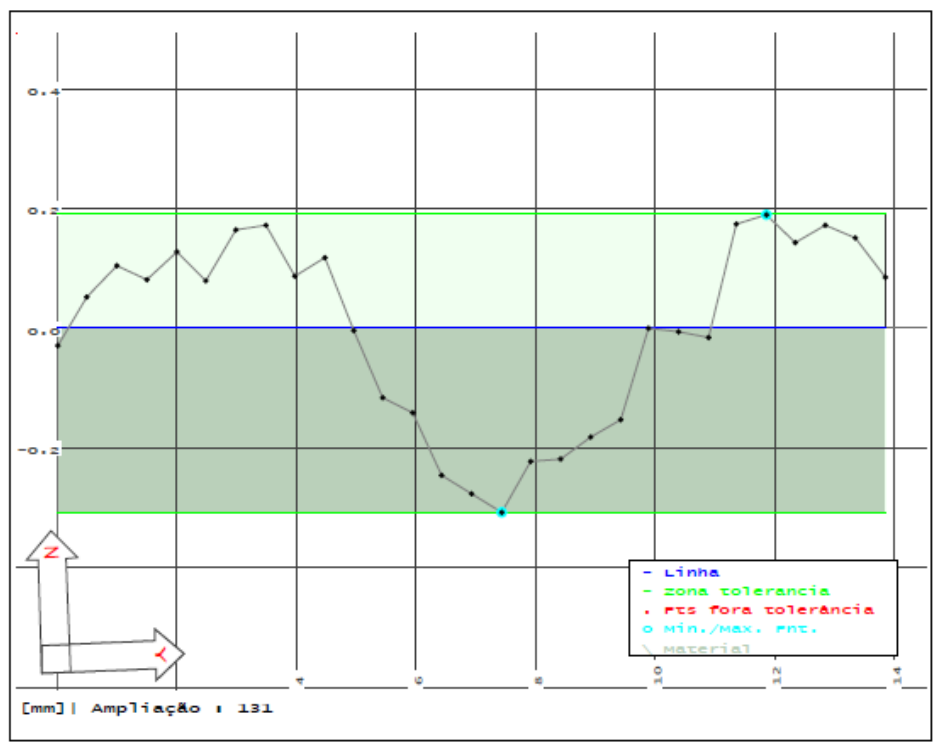

(C)

Figura 5: Gráficos representativos do ensaio de retilineidade, com limite de tolerância de $0,5 \mathrm{~mm}$ das amostras com diferentes tempos de tratamento térmico: (A) 10 minutos, (B) 25 minutos, (C) 55 minutos.

A partir da observação dos gráficos gerados pelo Software Genin, foi realizada a contagem dos pontos de picos e vales nas superfícies das amostras. Para a discussão dos ensaios, os pontos que estão no meio da linha base são relacionados as amostras que apresentam menor rugosidade, constatou-se então que em relação ao lado rugoso da amostra, a amostra que apresentou menos rugosidade foi a amostra $\mathrm{C}$, com 1 ponto na linha base, sendo 15 picos e 13 vales. Esse comportamento da amostra $C$ já era esperado devido a presença do $\mathrm{NaCl}$ encapsulado em sua superfície rugosa dificultando assim, na sua remoção no processo de lixiviação de partículas e tornando-a menos rugosa.

As demais amostras não apresentaram pontos na linha base, apresentando-se mais rugosas devido a uma maior remoção do $\mathrm{NaCl}$. No caso da amostra A ocorreu o arraste do polímero juntamente ao $\mathrm{NaCl}$ após a lixiviação de partículas. Nenhuma amostra avaliada apresentou pontos fora dos intervalos de tolerância. Foi possível observar também que a quantidade de picos e vales de todas as amostras apresentou-se bastante equilibrada. Na Tabela 1 é apresentada a sequência das diferentes amostras com sua quantidade de pontos nos gráficos. 
Tabela 1: Número de pontos das amostras com diferentes tempos de processamento.

\begin{tabular}{c|c|c|c|c|c}
\hline \multirow{2}{*}{ Amostra } & \multicolumn{5}{|c}{ Número de Pontos } \\
\cline { 2 - 6 } & Picos & Vales & $\begin{array}{c}\text { Linha Ba- } \\
\text { se }\end{array}$ & $\begin{array}{c}\text { Fora da } \\
\text { Tolerância }\end{array}$ & Pontos Totais \\
\hline A & 16 & 13 & - & - & 29 \\
\hline B & 15 & 14 & - & - & 29 \\
\hline C & 15 & 13 & 1 & - & 29 \\
\hline
\end{tabular}

\subsection{Espectroscopia na região do infravermelho com transformada de Fourier (FTIR)}

A Figura 6 apresenta os espectros na região do infravermelho para amostra controle e superfície rugosa da amostra. Como os resultados não apresentaram diferenças significativas entre os três diferentes tempos de processamento, para efeito de apresentação dos resultados, foi apresentado um gráfico que ilustra os resultados para ambos os tempos de processamento.

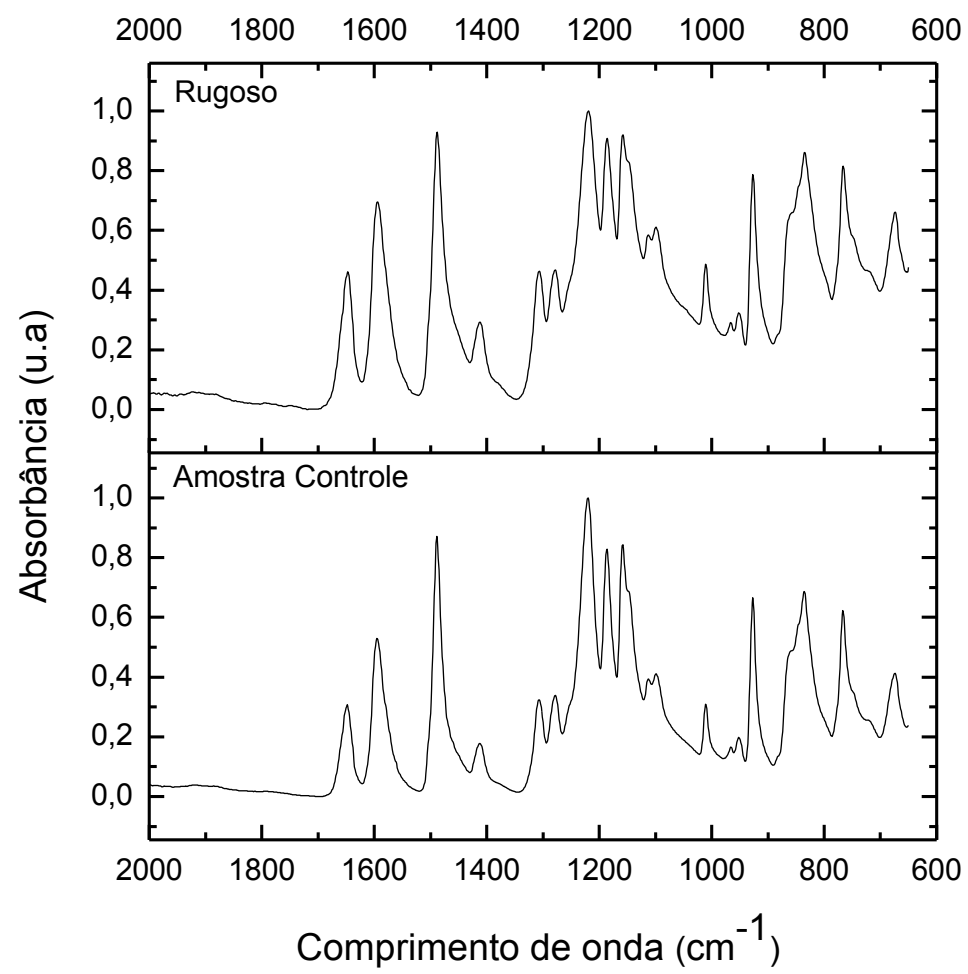

Figura 6: FTIR para as amostras de PEEK na superfície rugosa e na amostra controle.

Analisando os espectros de FTIR do PEEK da amostra controle e das amostras após processamento, na superfície rugosa foram detectadas bandas típicas de absorção em 675, 765 e $835 \mathrm{~cm}^{-1}$ apresentando deformação angular de grupos C-H aromáticos. Em 1157, 1185 e $1218 \mathrm{~cm}^{-1}$ estiramento assimétrico do grupo éter C-O. Nos diferentes espectros de PEEK foi observado que a banda mais intensa é a de $1218 \mathrm{~cm}^{-1} \mathrm{corres}^{-}$ pondente à estrutura de éter aromático. São encontradas também, bandas em 1278, 1306 e $1489 \mathrm{~cm}^{-1} \mathrm{com}^{-}$ vibrações de deformação axial referentes ao $\mathrm{C}=\mathrm{C}$ associado ao grupo $\mathrm{C}-\mathrm{O}$ éter aromático. Foi observado bandas de absorção em $1306 \mathrm{~cm}^{-1}$ e $1278 \mathrm{~cm}^{-1}$, associadas com o grupo carbonila da ligação cetona e a ressonância do grupo di-fenil éter, respectivamente, apresentando bandas características do PEEK. As bandas em 1594 e $1648 \mathrm{~cm}^{-1}$ correspondem ao estiramento principal e secundário da carbonila $\mathrm{C}=\mathrm{O}$. Estes resultados são similares aos encontrados na literatura [4, 21, 22, 23].

A partir da análise dos espectros observa-se que as amostras de PEEK, após os diferentes tempos de tratamento térmico quando comparadas a amostra controle, apresentaram um comportamento similar sem 
alterações significativas no perfil entre os materiais estudados.

\section{CONCLUSÕES}

Os resultados apresentados nesse estudo demonstram que houve uma efetiva modificação na superfície pela técnica de compressão, tratamento térmico seguido de lixiviação de partículas, obtendo assim estruturas com superfície modificadas. O aumento no tempo de tratamento térmico conduziu a uma melhor conformação e preservação das cavidades presentes na superfície rugosa como consequência de uma melhor fusão do polímero e lixiviação das partículas. O tempo excessivo de tratamento térmico, possivelmente, promoveu um processo oxidativo além de ocasionar uma menor efetividade na lixiviação devido ao processo de encapsulamento do $\mathrm{NaCl}$, promovendo assim, uma menor lixiviação. Em decorrência disto, a amostra que se apresentou menos rugosa foi a que possuiu um maior tempo de tratamento térmico.

Com base nas conclusões anteriores podemos dizer que a amostra B foi a que apresentou maior remoção de $\mathrm{NaCl}$ no processo de lixiviação, cavidades com tamanhos ideais para promover uma regeneração óssea adequada e maior rugosidade na superfície modificada.

\section{AGRADECIMENTOS}

Os autores agradecem ao apoio financeiro da Coordenação de Aperfeiçoamento de Pessoal de Nível Superior/CAPES, ao Laboratório de Avaliação e Desenvolvimento de Biomateriais do Nordeste (CERTBIO) e seus colaboradores, a OPAS (Organização Pan-Americana da Saúde) e a Universidade Federal de Campina Grande.

\section{BIBLIOGRAFIA}

[1] SIDDIQ, A.R., KENNEDY, A.R., "Porous poly-ether ether ketone (PEEK) manufactured by a novel powder route using near-spherical salt bead porogens: Characterisation and mechanical properties", Materials Science and Engineering, v.47, pp.180-188, 2015.

[2] MA, R., TANG, T., "Current strategies to improve the bioactivity of PEEK", International Journal of Molecular Sciences, v.15, pp.5426-5445, 2015.

[3] KURTZ, S.M., DEVINE, J.N., "PEEK Biomaterials in trauma, orthopedic, and spinal implants", Biomaterials, v.28, pp.4845-4869, 2007.

[4] KURTZ, S. M., Peek Biomaterials Handbook, $1^{\text {a }}$ ed., Waltham, PDL, 2012.

[5] TOTH, J.M., WANG, M., ESTES, B.T., et al., "Polyetheretherketone as a biomaterial for spinal applications", Biomaterials, v.27, pp.324-334, 2006.

[6] SCHWITALLA, A.D., EMARA, M.ABOU., SPINTING, T., et al., "Finite element analysis of the biomechanical effects of PEEK dental implants on the peri-implant bone", Journal of Biomechanics, v.48, pp.17, 2015.

[7] EVANS, N.T., TORSTRICK, F.B., LEE, C.S.D., et al., "High-strength, surface-porous polyether-etherketone for load-bearing orthopedic implants", Acta Biomaterialia, v.13, pp.159-167, 2015.

[8] DURHAM, J. W., RABIEI, A., "Deposition, heat treatment and characterization of two layer bioactive coatings on cylindrical peek", Surface and coatings technology, v.301, pp. 106-113, 2015.

[9] WIACEK, A. E., TERPILOWSKI, K., JURAK, M., et al., "Effect of low-temperature plasma on chitosan-coated PEEK polymer characteristics", European Polymer Journal, v.78, pp.1-13, 2016.

[10] GATTI, M. C. A., SILVA, R. V., TARPANI, J. R. "Análise térmica do laminado PEEK/carbono submetido a diferentes rotas de processamento", Revista Matéria, v.11, pp.332-339, 2006.

[11] REGO, B.T., Avaliação do comportamento mecânico de nanocompósito de PEEK/nHA sob curta e longa duração para aplicação como biomaterial, Dissertação de Mestrado, Universidade Federal de São Carlos, São Carlos, SP, Brasil 2012.

[12] SHRAVAN, S.P., MANGESH S.P, VINAYA, V.P., et al., "Polyaryletherketone biomaterial for use in medical implant applications", International Journal of Scientific and Research Publication, v.5, pp.44-49, 2015.

[13] TOTH, J.M., WANG, M., ESTES, B.T., et al., "Polyetheretherketone as a biomaterial for spinal applications", Biomaterials, v.27, pp.324-334, 2006. 
[14] WALSH, W.R., BERTOLLO, N., CHRISTOU, C., et al., "Plasma-sprayed titanium coating to polyetheretherketone improves the bone-implant interface", The Spine Journal, v.15, pp.1041-1049, 2015.

[15] BRYDONE, A.S., MEEK, R.D.M., DALBY, M.J., et al., Enhanced osteogenesis on PEEK polymer using injection mold nanopatterning, In: $2^{\mathrm{ND}}$ International PEEK Meeting. Washinhgton (USA), 2015.

[16] REIGNIER, J., HUNEAULT, M.A., "Preparation of interconnected poly( $\varepsilon$-caprolactone) porous scaffolds by a combination of polymer and salt particulate leaching", Polymer, v.47, pp.4703-4717, 2006.

[17] JONES, D.P., LEACH, D.C., MOORE, D.R., "Mechanical properties of poly(ether-etherketone) for engineering applications", Polymer, v.26, pp.1385-1393, 1985.

[18] DAY, M., SALLY, D., WILES, D.M., "Thermal degradation of poly(aryl-ether-etherketone): experimental evaluation of crosslinking reactions" Journal of Applied Polymer Science, v.40, pp.1615-1625, 1990.

[19] JONAS, A., LEGRAS, R., "Termal stability and crystallization of poly(aryl ether ether ketone)", Polymer, v.32, pp.2691-2706, 1991.

[20] WHANG, K., TSAI, D. C., NAM. E. K., et al., "Ectopic bone formation via rhBMP-2 delivery from porous bioabsorbable polymer scaffolds", Journal of Biomedical Materials Research, v.42, pp.491-499, 1998.

[21] NAKAMURA, H., NAKAMURA, T., NOGUCHI, T., et al., "Photodegradation of PEEK sheets under tensile stress", Polymer Degradation and Stabilit, v.91, pp. 740-746, 2006.

[22] MAZUR, R.L. Obtenção e caracterização de compósitos de fibras de carbono/PEEK com aplicações aeronáuticas, Tese de Doutorado, Universidade Estadual Paulista, Guaratinguetá, SP, Brasil 2010.

[23] SILVA, A. J. J., COSTA, M. F., Estudo e caracterização das propriedades quimiomecânicas do poli(éter éter cetona) (PEEK) envelhecido em álcool anidro. Painel PEMM PEMM/COPPE/UFRJ, Rio de Janeiro.

Brasil. 2011. 